\section{Does MOVES move the needle?}

Osteoarthritis (OA) of the knee is a common and vexing condition. Additional safe and effective modalities to treat this chronic condition are badly needed.

The article by Hochberg et al ${ }^{1}$ summarises the findings from the Multicentre Osteoarthritis interVEntion trial with SYSADOA (MOVES) study and concludes that the combination of glucosamine and chondroitin has equal efficacy to celecoxib, based on a 6-month, non-inferiority design using the change in the Western Ontario and McMaster osteoarthritis index (WOMAC) score as the assessment. Indeed, the combination product may be comparable to celecoxib as tested-the problem is that there is no certainty that celecoxib itself 'worked'.

The predicate study, ${ }^{2}$ called the Glucosamine/chondroitin Arthritis Intervention Trial (GAIT) study, compared celecoxib with glucosamine, chondroitin and the combination in a similar design, but included a placebo arm. GAIT failed to show a difference for any treatment in the change from baseline, as scored by the WOMAC instrument, compared with placebo, including celecoxib. The GAIT study confirmed, however, significant regression of scores in all groups over the 24 weeks of treatment, including placebo. While there was an apparent treatment response with the supplement combination in those patients with more severe WOMAC scores at baseline, this was an exploratory analysis. The study was not designed to specifically test for effects in this more severe subgroup, leading the authors to conclude that this observation was hypothesis-generating, not conclusive.

Having a proven treatment effect of the active comparator is the sine qua non of a non-inferiority design. None exists in this setting. Since the MOVES study did not incorporate a placebo arm, it is impossible to know whether there was assay sensitivity in this study-that is, that the study as conducted could have shown a difference were one to have existed. MOVES as designed provides no confidence that a treatment effect with celecoxib would have been evident despite the more severe population enrolled. It is entirely possible, if not probable, that the failure to show a difference between celecoxib and the supplement combination is because neither would have outperformed placebo. Unfortunately, despite the author's conclusion of comparable 'efficacy', there may be no efficacy at all.

MOVES unfortunately adds little to the understanding of the potential value of glucosamine and chondroitin use in OA of the knee, and the paper's conclusion is scientifically flawed.

\section{Robert Meyer}

Correspondence to Dr Robert J Meyer, Public Health Sciences, Virginia Center for Translational and Regulatory Sciences (VCTRS), Rm. 3231, West Complex MSB, PO Box 800717, Charlottesville, VA 22908-0717, USA; rjm7cd@virginia.edu

Handling editor Tore K Kvien

Twitter Follow Robert Meyer at @bobmeyer0719

Competing interests Small equity interest in Pfizer, which was the original manufacturer of celecoxib.

Provenance and peer review Commissioned; internally peer reviewed.

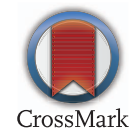

To cite Meyer R. Ann Rheum Dis 2015;74:e35.

Received 26 January 2015

Revised 2 February 2015

Accepted 8 February 2015

Published Online First 24 February 2015

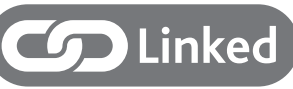

http://dx.doi.org/10.1136/annrheumdis-2015-207426

Ann Rheum Dis 2015;74:e35. doi:10.1136/annrheumdis-2015-207357

\section{REFERENCES}

1 Hochberg MC, Martel-Pelletier J, Monfort J, et al. Combined chondroitin sulfate and glucosamine for painful knee osteoarthritis: a multicentre, randomised, double-blind, non-inferiority trial versus celecoxib. Ann Rheum Dis 2015. Published Online First: 14 Jan 2015. doi:10.1136/annrheumdis-2014-206792

2 Clegg DO, Reda DJ, Harris CL, et al. Glucosamine, chondroitin sulfate, and the two in combination for painful knee osteoarthritis. N Engl J Med 2006;354:795-808. 